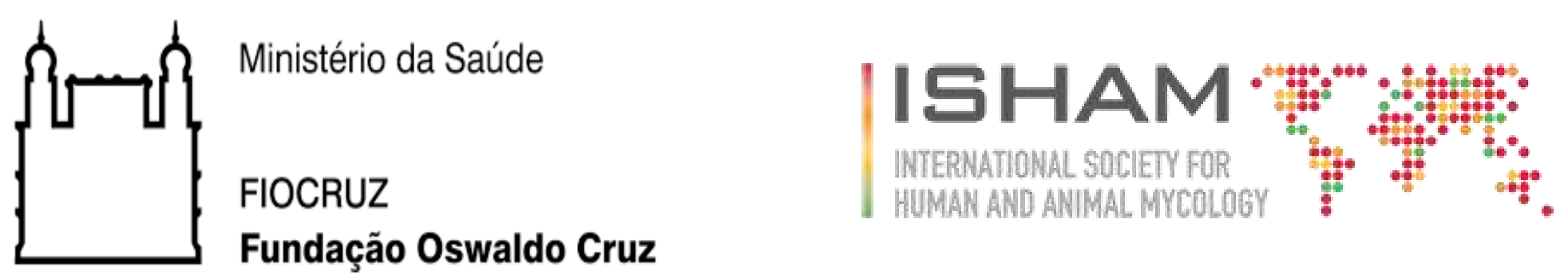

\title{
Feline sporotrichosis: the early treatment as a key factor to control the zoonotic transmission
}

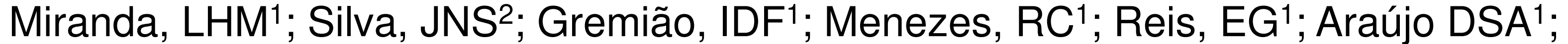 \\ Machado, ACS ${ }^{1}$; Boechat JS ${ }^{1}$, Ferreiro, L'ㄹ Pereira, SA $^{1}$
}

\section{1 - Laboratório de Pesquisa Clínica em Dermatozoonoses em Animais Domésticos, Instituto Nacional de Infectologia Evandro Chagas (INI), Fundação Oswaldo Cruz (Fiocruz)}

2 - Laboratório de Micologia, Faculdade de Veterinária, Universidade Federal do Rio Grande do Sul (UFRGS)

Background Feline sporotrichosis is frequently related to skin lesions with high loads of yeast-like cells, making the cat an important source of infection. Despite the importance of cats in the zoonotic transmission of Sporothrix, the dynamics of the fungal burden after the onset of antifungal treatment is unknown.

Objective The aim of this study was to compare the dynamics of the fungal load in cats with sporotrichosis during treatment with itraconazole (ITZ) as a monotherapy or combined with potassium iodide $(\mathrm{KI})$

Methods One hundred and thirty cats with sporotrichosis presenting skin ulcers were divided into two groups: Group 1 $(\mathrm{n}=74)$ was treated with ITZ $100 \mathrm{mg} / \mathrm{cat} /$ day and group $2(\mathrm{n}=56)$ with ITZ $100 \mathrm{mg} / \mathrm{cat} /$ day and KI $2.5 \mathrm{mg} / \mathrm{kg} /$ day. The largest skin ulcer from each cat was followed up monthly through cytopathological assessment of fungal load (impression smears stained by Quick Panoptic method) until complete healing or up to 12 weeks. The fungal load was obtained by the means of yeast-like cell counts from three 1000x magnification fields per sample.

Results In both groups 1 and 2, most of the evaluated lesions (56.8\% and $78.6 \%$, respectively) healed during the first month of treatment. A significant decrease on the fungal load over the treatment occurred between the first visit (before the beginning of the treatment) and the first follow up in groups 1 and 2 ( $p<$ $0.001 ; p<0.001$, respectively) and between the first and second follow up in group $1(p=0.003)$. After 12 weeks of treatment, 62 cats $(83.8 \%)$ from group 1 had the evaluated skin ulcer healed. The persistence of the lesion on the other 12 cats after this period was related to higher fungal loads $(p=0.013)$ before the beginning of the treatment (Median=98.6), in comparison to lesions that have healed (Median=15.0). The median of the fungal load was 0.33 after 8 weeks of treatment in lesions that have not healed. In group 2, all evaluated lesions were healed at the $12^{\text {th }}$ week-appointment, thus the fungal load could not be assessed at this point. No correlation was detected between the groups concerning the fungal load at any time point. With regards to the treatment outcome, clinical cure occurred in 57 (77.0\%) animals from group 1. Three cats were lost to follow-up.
The fungal loads before and after one month of treatment were higher ( $p=0.022$ and $p<0.001$, respectively) on cats that failed to cure in comparison to those that were discharged in this group. Treatment failure was not observed in group 2: the cure rate was $94.6 \%$ and two cats (5.4\%) were lost to follow-up

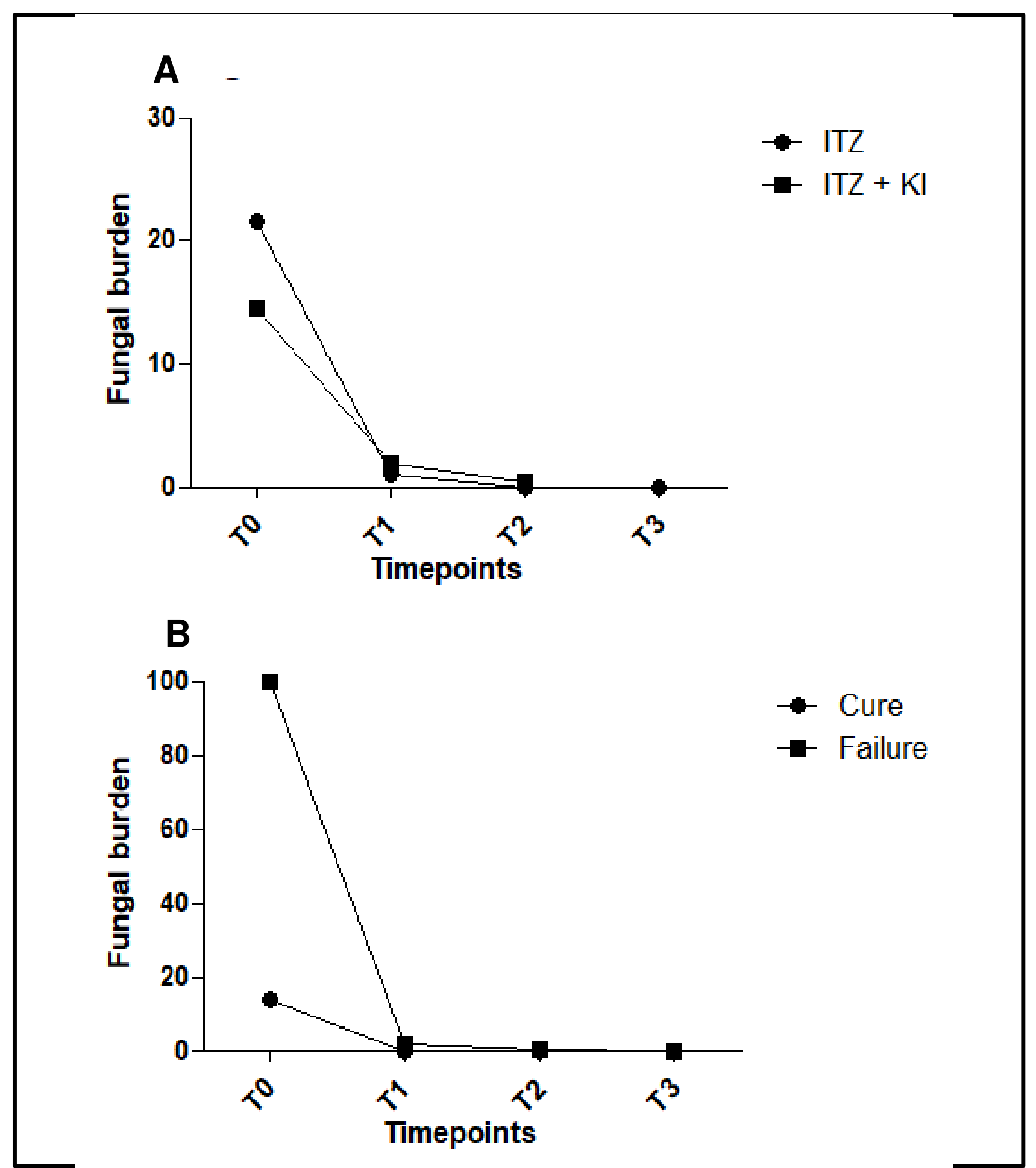

Figure 1. Fungal burden variation over treatment: (A) with Itraconazole alone (ITZ) or in association to $\mathrm{KI}(\mathrm{ITZ}+\mathrm{KI})$; (B) according to clinical outcome after treatmen with ITZ.

Conclusions There was a remarkable decrease of fungal load taking place early during the therapy on both groups, even in cases that required longer length of antifungal treatment as well as in cases that failed to cure. The reduced fungal load in skin lesions from treated cats might represent a decreased risk of zoonotic transmission. Still, the higher fungal load before the outset of the treatment is likely to increase the chance of therapeutic failure with ITZ alone. On the other hand, the association of $\mathrm{KI}$ to ITZ showed a high cure rate, with the treatment outcome not being influenced by the fungal load. Therefore, we highly recommend this protocol as a promising alternative for the treatment of feline sporotrichosis 